\title{
Heartbeat: Highlights from the issue
}

There are so many interesting articles in each issue of Heart that it is a challenge to pick just a few to highlight in Heartbeat. Some are mentioned here with several other articles you may want to read shown in the table of contents.

B-type natriuretic peptide (BNP) has favorable hemodynamic effects in heart failure patients but also increases the risk of ventricular arrhythmias, negating the potential benefits of intravenous administration of this therapy. In this issue of Heart, Prof Thireau (see page 833) and colleagues hypothesized that the combination of subcutaneous BNP and a selective beta 1 -adrenergic blocker would "unmask" the beneficial effects of BNP by blocking the associated adverse adrenergic effects. This hypothesis was tested in a murine model of ischemic heart failure with results showing that combined therapy reduced fibrosis and hypertrophy and improved cardiac function in association with a decreased occurrence of ventricular arrhythmias, compared to $\beta$-blocker therapy alone.

In the accompanying editorial, Prof ElArmouche and colleagues (see page 819) provide insight into the "complex interplay between the classical neurohormonal pathways and diverse biologically active peptides during heart failure progression" and suggest that understanding this interplay might provide new targets for therapy in heart failure patients. The editorial puts the data from the experimental model into the context of clinical trials of BNP therapy. The authors highlight the "known unknowns" and illustrate how the intriguing findings by Prof Thireau and colleagues may lead to novel therapeutic targets in patients with heart failure (see figure 1).

The topic of nonadherence to medical therapy is addressed again in this issue in a study of hypertensive patients by Dr. Tomaszewski and colleagues (see page 855). Using high-performance liquid chromatography-tandem mass spectrometry urine analysis to screen for 40 antihypertensive medications, they found that $25 \%$ of patients were not fully adherence with the prescribed anti-hypertensive therapy. Lack of adherence to therapy was linearly related to blood pressure and was associated with inadequate blood pressure control (see table 1).

Correspondence to Professor Catherine M Otto Division of Cardiology, University of Washington, Seattle, WA 98195, USA; cmotto@u.washington.edu

Paulus Kirchhof and Catherine M Otto

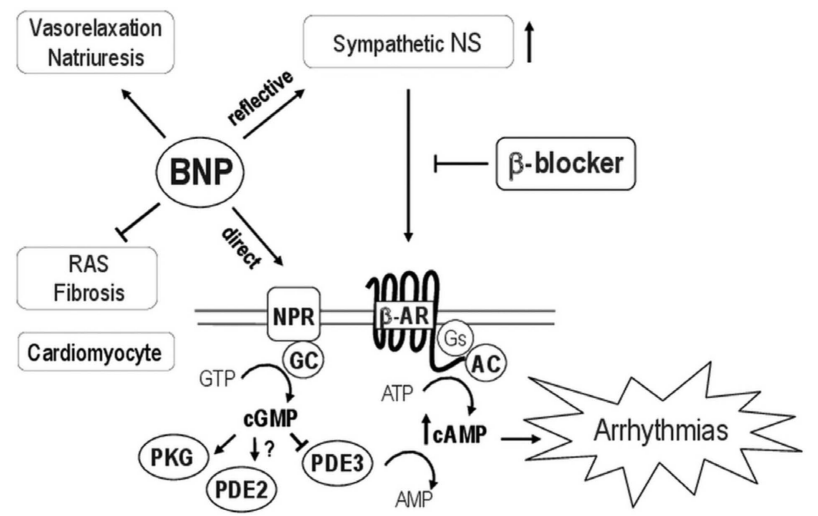

Figure 1 Schematic figure illustrating the brain natriuretic peptide (BNP)-dependent pathways and the possible interactions with $\beta$ blocker treatment. cGMP, cyclic guanosine monophosphate, CAMP, cyclic adenosine monophosphate, NPR, natriuretic peptide receptor.

Professor Brown (see page 821) notes although about $20-25 \%$ of patients with "resistant" hypertension have primary aldosteronism, the study by Dr. Tomaszewski and colleagues suggests that the remainder may simply not be taking their medications. He suggests that more widespread use of the mass spectrometry assay could transform practice by providing accurate information about medication adherence and prevent unnecessary additional procedures, such as renal denervation, when "resistant" hypertension is really just non-adherence. He concludes: "Philosophers define knowledge as belief

with evidence. No longer need we guess whether patients have resistant hypertension. We can know."

The use of statins in for prevention of new or recurrent coronary events is a topic of current interest with the recent release of the JBS3 prevention guidelines as well as the ACC/AHA recommenations. In a large retrospective longitudinal study, Prof Lang and colleagues (see page 867) found that the adjusted hazard ratio for mortality was 0.72 (95\% CI 0.59 to 0.88 , $\mathrm{p}<0.001)$ for those taking a high potency statin compared to those taking simvastatin. The association between high potency

Table 1 Association between blood pressures and non-adherence to antihypertensive treatment among hypertensive patients

\begin{tabular}{|c|c|c|c|c|c|c|c|}
\hline $\begin{array}{l}\text { Blood } \\
\text { pressure }\end{array}$ & Adherent & $\begin{array}{l}\text { Any } \\
\text { non-adherent }\end{array}$ & $\begin{array}{l}\text { Complete } \\
\text { non-adherent }\end{array}$ & $\begin{array}{l}\text { Beta } \\
(\mathrm{SE})^{*}\end{array}$ & p Value* & $\begin{array}{l}\text { Beta } \\
(\mathrm{SE}) \dagger\end{array}$ & p Valuet \\
\hline Clinic SBP $\ddagger$ & $161 \pm 24.0$ & $170 \pm 24.7$ & $177 \pm 28.5$ & $-9(3.7)$ & 0.0209 & $-18(5.4)$ & 0.0010 \\
\hline Clinic DBP & $90 \pm 14.4$ & $100 \pm 19.1$ & $107 \pm 18.3$ & $-9(2.4)$ & 0.0003 & $-16(3.5)$ & $1.0 \times 10^{-5}$ \\
\hline $\begin{array}{l}24 \mathrm{~h} \text { daytime } \\
\text { SBP§ }\end{array}$ & $152 \pm 19.8$ & $159 \pm 21.1$ & $165 \pm 17.3$ & $-6(4.2)$ & 0.1814 & $-14(5.5)$ & 0.0146 \\
\hline $\begin{array}{l}24 \mathrm{~h} \text { daytime } \\
\text { DBP§ }\end{array}$ & $86 \pm 13.1$ & $94 \pm 13.0$ & $100 \pm 9.8$ & $-6(2.6)$ & 0.0286 & $-11(3.2)$ & 0.0006 \\
\hline
\end{tabular}

*Difference between adherence and any non-adherence (both partial and complete) after adjustment for age, sex, ethnicity and clinical category.

tDifference between adherence and complete non-adherence after adjustment for age, sex, ethnicity and clinical category.

¥Systolic blood pressure (SBP) and diastolic blood pressure (DBP) -information available for 152 adherent, 52 any non-adherent and 21 completely non-adherent patients.

$\S 24 \mathrm{~h}$ daytime SBP and DBP-information available for 121 adherent, 26 any non-adherent and 15 completely non-adherent patients, data in columns 2-4 are means and SDs of absolute blood pressure values recorded by clinic measurements and $24 \mathrm{~h}$ ambulatory blood pressure monitoring. Beta- $\beta$-coefficient, $\mathrm{p}$ value-level of statistical significance; all from adjusted linear regression models with blood pressure as dependent quantitative variable and age, sex, ethnicity and clinical category (new referrals, follow-up patients, referrals for renal denervation) as well as non-adherence to antihypertensive treatment as independent parameters included in the model. 
Table 2 Proportional hazards model for risk of death

\begin{tabular}{|c|c|c|c|}
\hline & HR & $95 \% \mathrm{Cl}$ & p Value \\
\hline \multicolumn{4}{|l|}{ Cohort (vs simvastatin monotherapy) } \\
\hline High-potency statin monotherapy & 0.72 & 0.59 to 0.88 & $<0.001$ \\
\hline Ezetimibe/statin combination & 0.96 & 0.64 to 1.43 & 0.847 \\
\hline Gender (female vs male) & 0.84 & 0.74 to 0.95 & 0.009 \\
\hline Age (per year) & 1.08 & 1.08 to 1.09 & $<0.001$ \\
\hline Smoker (yes vs no) & 1.44 & 1.25 to 1.65 & $<0.001$ \\
\hline Diabetic (yes vs no) & 1.44 & 1.13 to 1.83 & $<0.001$ \\
\hline Further MI during follow-up & 1.45 & 1.32 to 1.60 & $<0.001$ \\
\hline \multicolumn{4}{|l|}{ Cardiovascular drugs (yes vs no) } \\
\hline Aspirin & 0.57 & 0.48 to 0.69 & $<0.001$ \\
\hline$\beta$-Blockers & 0.68 & 0.59 to 0.79 & $<0.001$ \\
\hline ACE-I & 0.72 & 0.62 to 0.84 & $<0.001$ \\
\hline DHP CCB & 0.57 & 0.47 to 0.69 & $<0.001$ \\
\hline Non-DHP CCB & 1.06 & 0.84 to 1.32 & 0.624 \\
\hline Nitrate & 1.00 & 0.86 to 1.16 & 0.582 \\
\hline Statin compliance & 0.94 & 0.93 to 0.94 & 0.428 \\
\hline
\end{tabular}

statin use and fewer deaths was confirmed in a propensity score analysis taking into account covariates that predicted drug treatment group. In contrast, ezetimibe treatment was not associated with a lower mortality (see table 2).
In the accompanying editorial, Professors Halcox and Currie (see page 825 ) point out that this data lends further support to the concept that the clinical benefit of statin therapy are more closely related to the intensity of drug therapy rather than to the magnitude of reduction in serum low density lipoprotein (LDL) cholesterol levels. However, they caution that this retrospective study has several limitations and that results of ongoing randomized controlled clinical trials may modify these conclusions.

The Education in Heart article (see page 881 ) in this issue discusses potential clinical applications of magnetic resonance spectroscopy in patients with heart failure and ischemic heart disease. The Image Challenge (see page 892 ) shows an interesting angiographic finding and asks you to interpret the images - the answer provides some nice optical coherence tomographic images and 3D reconstructions to emphasize the key features of the diagnosis.

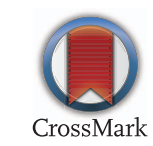

To cite Kirchhof P, Otto CM. Heart 2014;100:817818.

Heart 2014;100:817-818. doi:10.1136/heartjnl-2014-306041 